\title{
Inhibition of Elastin and Collagen Networks Degradation in Human Skin By Gingival Fibroblast. in Vitro, Ex Vivo and in Vivo Studies
}

\author{
Bruno Gogly ${ }^{\mathrm{a}, \mathrm{b}}$, François Côme Ferré ${ }^{\mathrm{a}, \mathrm{b}}$, Hafida Chérifi ${ }^{\mathrm{a}}$, Adrien Naveau ${ }^{\mathrm{a}, \mathrm{b}}$, Benjamin Philippe \\ Fournier $^{\mathbf{a}, \mathbf{b}}$
}

${ }^{a}$ Dept. of dentistry, A.Chenevier-H. Mondor hospital. AP-HP, Créteil, France; ${ }^{b}$ Centre de recherches des Cordeliers. Pierre et Marie Curie University, Paris, France. Paris-Descartes University UMR S872, Paris, France. INSERM, U872, Paris, France Email: bruno.gogly@ach.aphp.fr

Received February 25 $5^{\text {th }}, 2011$; revised March 20 $0^{\text {th }}, 2011$; accepted March $25^{\text {th }}, 2011$.

\begin{abstract}
Skin aging shows an imbalance between synthesis and degradation of the extracellular matrix. The overproduction of degradative enzymes (MMPs) during the chronology-and photo-induced aging leads to a degradation of the elastic and collagen networks. In a model of collagen and elastin destruction, we showed that the gingival fibroblast was able to preserve these macromolecules by inhibiting the overproduction of metalloproteinases by overproduction of TIMP-1 and modulation of the inflammatory cytokines activity. The objective of this study is to evaluate the effect of the gingival fibroblasts on human skin. The results in vitro and ex vivo show that the gingival fibroblast protects the skin collagen and elastic network by the inhibition of MMPs which leads to an overproduction of the TIMP-1. Moreover, the gingival fibroblast modulates the activity of some enzymes responsible for the inflammation; they inhibit the IL-1 $\beta$ and stimulate the production of TGF- $\beta 1$. In vivo studies with a duration of six months and 50 women with pronounced wrinkles show that the culture supernatant of gingival fibroblasts diluted to $5 \%$ leads to a statistically significant decrease in the number and length of wrinkles.
\end{abstract}

Keywords: Elastin, Collagen, Skin, Matrix Metalloproteinases, Gingival Fibroblast

\section{Introduction}

In skin ageing, a disequilibrium occurs in the balance between the synthesis of the extracellular matrix (ECM) and its degradation by matrix metalloproteases (MMPs). This disequilibrium leads to an excessive degradation of the extracellular matrix, a characteristic of skin ageing [1]. Skin ageing is associated to an increase of the number and the deepness of wrinkles, a direct consequence of the degradation of macromolecules of the dermis, such as collagens and elastin. In dermis, the MMP overproduction, which occurs in chronological and photo-induced ageing, is stimulated by oxygenated free radicals. Besides, in the skin areas exposed to sun, such as facial skin, other deleterious effects of UV rays occur, in particular an incomplete collagen synthesis, a skin pigmentation, and a solar elastosis (which appears as a degradation of the cutaneous elastic lattice). Furthermore, in vitro studies have shown that MMP are overproduced by skin fi-broblasts submitted to UV-ray treatment [2]. Collagenases-1 (MMP-1) degrade collagens, while gelatinases A and B (MMP-2 and MMP-9) degrade elastin. Other metalloproteinases, such as stromelysin 1 (MMP-3), are involved both in the collagen and elastin degradation. It is evident that the activity of the MMPs is one of the keys of the skin ageing and that these enzymes have thus to be the target of therapeutic activities $[3,4]$.

Oral mucosal wounds heal with a minimal scarring. The gingival tissue surrounding the teeth contains an unique connective tissue cell type, gingival fibroblasts, that contribute to a scarless healing in the oral mucosa. A scarless healing of oral mucosa is characterized by a faster resolution of inflammation and the control of myofibroblast action compared. [5,6]

Our last works show that the gingival fibroblast in coculture with arteries inhibits the activity of the MMP-1, MMP-3, MMP-7 and MMP-9 [7-9]. This inhibition is 
due to the over expression of the TIMP-1, the inhibitor of these enzymes, and TGF- $\beta 1$ particularly mattering to fight against the skin ageing. Besides, this inhibition property of the MMPs is specific of the gingival fibroblast. Indeed, the dermal and adventitial fibroblasts, in the presence of arteries, have no effect on these enzymatic activities [7]. Finally the vascular consequence of this inhibition capacity of the MMPs by the gingival fibroblast is translated into a protection and a conservation of the arterial elastic network. It thus seems particularly interesting to study the capacity of protection of the cutaneous macromolecules by the gingival fibroblast. We thus realized in vitro and ex vivo experiments on models of cellular and cutaneous ageing by UVA irradiations. We studied the effects of the GF on the activity of the MMPs by the dermic fibroblasts and the skin as well as the conservation of the elastin and the cutaneous collagen. Finally we realized a clinical study, on 50 women, on the decrease of the crows' feet by a cream containing $5 \%$ of culture medium supernatant of gingival fibroblasts after having tested the toxicity of this asset.

\section{Material \& Methods}

\subsection{Organotypical Ex Vivo Experiments}

Five human skin biopsies cultures were obtained from normal subjects after aesthetic or reconstructive surgery. All biopsies were rinsed with a Hank's solution. Each of the five skins samples was split up into fragments of 5 $\mathrm{mm}$ with a trocar, each fragment was weighed and UVA-irradiated (or not) at 15 joules/cm2 $\left(\mathrm{Si}_{15}\right)$ and then cultivated in a collagen with (or without) human gingival fibroblast (hGF) or dermal fibroblast (hDF). All the details of the cells obtaining are explained in the in vitro cellular experiments

In a $10 \mathrm{ml}$ Erlenmeyer, $6 \mathrm{ml}$ of serum free medium (SFm) or $6 \mathrm{ml}$ of SFm containing $150000 \mathrm{hGF}$ or $\mathrm{hDF}$ (see the paragraph in vitro cellular experiments), $3.4 \mathrm{ml}$ of rat type I collagen (Jacques Boy Institute, $2 \mathrm{mg} / \mathrm{ml}$, ref: 207050257) and $600 \mu \mathrm{l}$ filtered $\mathrm{NaOH} 0.1 \mathrm{~N}$ (to neutralize the acid collagen solution) were mixed. Then a skin fragment (with or without $150000 \mathrm{hGF}$ or hDF) was deposited in the prepared solution and carefully homogenized with the solution. Lastly, the whole was placed in a $60 \mathrm{~mm}$ Petri dish and in an incubator at $37^{\circ} \mathrm{C} / 5 \% \mathrm{CO}_{2}$.

Five control collagen lattices were cultivated without skin fragments, in order to test whether bioactive molecules within the collagen gel and the culture medium would contribute to the results.

The cultures were maintained for a week and analysed at day 1, 3 and 7. At each point of the experimentation 3 $\mathrm{mL}$ of supernatant were collected and stored at $-80^{\circ} \mathrm{C}$ for further analysis of the secreted proteins.

\subsection{Histological Analysis by Computerized Image Analysis}

The skin biopsies (control at day 0 ) and their surrounding collagen lattices were collected after 3 (data not shown) and 7 days, rinsed with PBS 1x and fixed in Paraformaldehyde 4\%/PBS during 48 hours. They were then dehydrated with alcohol $70 \%$, then $95 \%$, then $100 \%$ and finally in a toluene before a paraffin inclusion. Sections (7 $\mu \mathrm{m})$ were stained using three different specific protocols.

Hemalun/eosin protocol: after rehydration, the sections were covered with hemalun during five minutes before using tap water, then eosin during 1 minute.

Sirius Red protocol (collagen network): after rehydration, the sections were covered with Sirius Red during 30 minutes. The quantification of collagen density was performed within 2 steps. Firstable, the colour image was transformed into monochrome with a 255 level grey scale. Thereafter, we evaluated the relative number of pixels classified as red by adjusting the threshold permitting a binary analysis. The measurements were performed in three independent fields by slide.

Orcein protocol (elastin network): after partial rehydration (stopped with alcohol 95\%), the sections were immersed into an orcein solution during half an hour. A fast rinsing with alcohol 95\% (with 2 hydrochloric acid drops added) was then carried out. Slides were lastly rinsed with distilled water before dehydratation and final assembling. The elastin density was quantified similarly as the collagen density.

\subsection{Protein Analysis}

MMP-2 and MMP-9 were analysed by gelatin zymography, MMP1 by collagen zymography and MMP3 by casein zymography [10]. Ten microliter of culture medium were analyzed for each sample. Ten microliters of proMMP-9 $(92 \mathrm{kDa})$ and $10 \mu \mathrm{l}$ of pro-MMP-2 $(72 \mathrm{kDa})$ (BC058 and $\mathrm{BC} 057$; $\mathrm{ABCys}$ ) were electrophoresed on the same gel in order to facilitate the identification of the MMP types.

MMP-1 (DMP100), MMP-2 (DMP200), MMP-3 (DMP300), MMP-9 (DMP900), TIMP-1 (DTM100), TIMP-2 (DTM200), IL-1 $\beta$ (DLB 50) and TGF- $\beta 1$ (DB100B) were analysed by ELISA (R\&D Systems) according to the recommendations of the manufacturer.

The statistical analysis was made using Wilcoxon and Mann-Whitney tests. Please note that the isoforms of these molecules are much conserved between the various species: it enabled the use of human ELISA kits for a human supernatant immunodetection. 


\subsection{Cellular in Vitro Experiments}

Five Human gingival fibroblast (hGF) cultures were obtained from gingival explants of healthy patients with no periodontitis history. Five Human dermal fibroblast (hDF) cultures were obtained from skin biopsies of normal subjects after aesthetic or reconstructive surgery (the same biopsies as those used for organotypic studies). All patients (20-30 years old) gave their informed consent according to the Helsinki Declaration (1975) and denied having recently taken drugs that could affect connective tissue metabolism. The tissue samples were divided into two parts: one for a histological analysis and the other for a cell culture. The serial gum tissue sections were stained with hematoxylin and eosin for an assessing of the tissue quality and the absence of inflammatory infiltrates in the gingiva. Only the histological healthy tissue was used for experiments.

The primary cultures were established in $25 \mathrm{~cm}^{2}$ culture flasks from explants and used from passage 3 to 5 . The monolayer cultures were maintained during 3 weeks in Dulbecco's modified Eagle's medium (DMEM, Invitrogen-Life Technologies, Cergy Pontoise, France) with Knock-Out Serum Replacement (SFM, Serum Free Medium) (KOSR, Invitrogen-Life Technologies) at $37^{\circ} \mathrm{C}$ in a $5 \% \mathrm{CO}_{2}$ and $95 \% \mathrm{O}_{2}$ atmosphere. The cell culture medium was changed every 72 hours. After the first passage, the cells were routinely maintained either in SFM.

The Serum-free medium (SFm) developed in our study consisted of DMEM supplemented with 20\% KOSR. The components of KOSR have been previously published [11]. The SFm was optimized with $1 \mathrm{mM}$ L-Glutamine and 1\% non-essential amino acids. The SFm required supplementation with basic Fibroblast Growth Factor (b-FGF, $1 \mathrm{ng} / \mathrm{ml}$, human recombinant; Invitrogen-Life Technologies) for long term culture, and insulin $(5 \mu \mathrm{g} / \mathrm{ml}$, human recombinant; Invitrogen-Life Technologies) for facilitating cell cycle progression.

\subsection{Preparation of hGF or hDF Conditioned Medium (hGFm or hDFm)}

The culture medium from $75 \mathrm{~cm} 2$ flasks of confluent hGF and hDF cultures, was discarded. $24 \mathrm{ml}$ of SFm was then added and retrieved 24 hours later. The conditioned medium (hGFm or $\mathrm{hDFm}$ ) was then frozen at $-20^{\circ} \mathrm{c}$ until use.

\subsection{Preparation of the cells}

Three 12-wells plates from each culture were seeded with $\mathrm{hDF}$ from two $25 \mathrm{~cm} 2$ flasks at confluence. When the confluence was reached $(150,000$ cells per well), 2 plates of hDF were UVA-irradiated respectively at 7.5
$\left(\mathrm{hDFi}_{7.5}\right)$ and $15\left(\mathrm{hDFi}_{15}\right)$ joules/cm2, the third plate was used as a control, in order to check the absence of MMP-9 in absence of irradiation. The culture media were changed after irradiation. For each flask, the following media were added: SFm only for 4 wells $(1 \mathrm{ml}$ per well), hGFm for 4 wells ( $1 \mathrm{ml}$ per well), hDFm for 4 wells ( 1 $\mathrm{ml}$ per well). The culture media were then collected 24 hours later, aliquoted and stored at $-80^{\circ} \mathrm{C}$ for a further protein secretion analysis.

\subsection{Human in Vivo Experiments}

We led a study on the human anti-wrinkle efficiency of the hGF conditionned media (hGFm). Firstable some studies of toxicity of hGFm were realized, before the testing of a neutral cream containing $5 \%$ of hGFm on fifty voluntary women for six months. $200 \mathrm{~mL}$ of hGFm were obtained from the five cultures between the passages 3 and 6 as described previously.

\subsection{Studies of Toxicity.}

All the studies were performed in compliance with the principles of the Good Laboratory Practice (G.L.P.), as defined in the directive 2004/10/EC, the O.E.C.D. ruling relative to the mutual acceptance of data in the evaluation of chemical substances.

\subsection{Mutagenic Potential of hGFm}

The bacterial reverse mutation test (Ames test) was performed in order to evaluate the mutagenic potential of hGFm. Suspensions of 5 amino-acid requiring strains of Salmonella typhimurium (TA98, TA100, TA102, TA1535, TA1537) were exposed by the direct plate incorporation method to five doses (from $5 \mu \mathrm{L}$ to $0.06 \mu \mathrm{L}$ ) of the test item in the presence and in the absence of an exogenous metabolic activation system. Both tests were repeated according to the pre-incubation method. The revertant bacteria due to point or frameshift-mutations at specific locus are able to grow, forming colonies. These colonies were counted and compared to the number of spontaneous revertant colonies on solvent control plate (negative control). Similarly, specific standard mutagens were tested and used as positive controls.

\subsection{Skin Sensitisation Potential of hGFm.}

A local Lymph Node Assay test was performed to assess the skin sensitisation potential of hGFm in the CBA/J strain mouse, following topical applications to the dorsal surface of the ear. Three groups, each of four animals, were treated for three consecutive days (D1, D2, D3) with $50 \mu \mathrm{L}(25 \mu \mathrm{L}$ per ear) of the undiluted test item and 
of the test item as a solution in dimethyl formamide (DMF) at a concentration of $25 \%$ and $50 \%(\mathrm{v} / \mathrm{v})$. A further group of four animals was treated with DMF. On D6, the proliferation of lymphocytes in the draining auricular lymph nodes was determined by cell counting.

\subsection{Skin Irritation Test}

The hGFm was applied at the dose of $0.5 \mathrm{ml}$, under semi-occlusive dressing during 4 hours on an undamaged skin area of three rabbit. The experimental procedure protocol was established from the O.E.C.D guideline $n^{\circ} 404$ dated April $24^{\text {th }}, 2002$ and the test method B.4 of the council regulation $\mathrm{N}^{\circ} 440 / 2008$.

\subsection{Eye Irritation Test}

The hGFm was instilled into the eye of three New Zealand rabbits at the dose of $0.1 \mathrm{ml}$. The experimental protocol was established on the basis of the official method defined in the O.E.C.D guideline $\mathrm{n}^{\circ} 404$ dated April $24^{\text {th }}$, 2002 and the test method B.5 of the council regulation $\mathrm{N}^{\circ} 440 / 2008$.

\subsection{Human Clinical Study of the Anti-Wrinkle Efficiency}

The clinical study was realized by the Institute of Dermatology of Aquitaine (IDEA) on fifty voluntary women from 45 to 50 years old and presenting pronounced wrinkles at the aera of the crow's-foot. The volunteers were informed and gave their consent. The clinical study aims at estimating the cutaneous tolerance and the anti-wrinkle effect of a cosmetic cream containing $5 \%$ of active product. The neutral cream containing $5 \%$ of hGFm was packaged in 200 flasks of $50 \mathrm{ml}$. The cosmetic product is applied in the morning and evening during six months to the outline of eyes by insisting on crow's-feet.

The evaluation of the anti-wrinkle potential is realized by the measure of the number of wrinkles, their total surface $(\mathrm{mm} 2)$, their total length $(\mathrm{mm})$, their average length $(\mathrm{mm})$ and their depth $(\mu \mathrm{m})$. The micro cutaneous relief is analyzed after imprint by analyses of images by the software Quantirides. The results are analyzed by the statistical test of Wilcoxon and are significant for $\mathrm{p}<0.05$.

\section{Results}

\subsection{Organotypic Ex Vivo Experiments}

The irradiated skin secretes more MMPs than the control skin and the co-culture with hGF decreases the MMPs levels more than the co-culture with hDF (Figure 1.a). The amount of MMP-1 is multiplied by 2.2, the MMP-3 and MMP-9 by 1.8 and 2.6 respectively. The quantity of MMP-2 remained stable in all experimental conditions.
The hGF in coculture with irradiated skin causes a decrease of the amount of MMP-1 (-1.7 fold), MMP-3 (-1.5 fold) and MMP-9 (-2.2 fold). There is a smaller decrease in coculture with the presence of hDF: -1.2 times respectively for MMP-1 and MMP-3 and -1.3 times for MMP-9.

The coculture with hGF and irradiated skin tends to normalize the concentration of cytokines in the culture medium (Figure 1.b). The irradiated skin secretes more IL-1 $\beta$, a major inflammation cytokine, and less of TGF$\beta 1$, a cytokine which appears as important in skin healing, in comparison with the control skin. At day 7 of culture, the irradiated skin secretes 2.5 times more IL- $1 \beta$ and 1.4 times less TGF- $\beta 1$. The co-culture with hGF can restore more physiological concentrations of these two cytokines. The concentration of IL-1 $\beta$ decreased 4.3 times compared to the irradiated skin and falls lower than the control skin. The concentration of TGF- $\beta 1$ becomes equivalent to the one of the control skin. The hDF has the same effect but to a lesser extend than hGF. The IL-1 $\beta$ decreases by 1.3 times with $\mathrm{hDF}$ compared to the irradiated skin and is 3.3 times higher with hGF than the control skin. TGF- $\beta 1$ increases by 1.2 times with hDF compared to the irradiated skin only and by 1.4 times in co-culture with Hgf.

The decrease of free forms of MMPs is due to the formation of a MMP/TIMP1 complex (Figure 1.c and Figure 1.d). Co-culture of irradiated skin with hGF secretes more TIMP-1 than with $\mathrm{hDF}$ (x2.5). The amount of MMP-1/TIMP-1 and MMP-3/TIMP-1 complexes is higher in the supernatant of irradiated skin compared to the control skin: the concentrations are respectively 1.5 times and 1.3 times increased. However, the difference is not statistically significant for the complex TIMP1/MMP-1.

The MMP-9/TIMP-1 complex was not statistically increased in irradiated skin after 7 days of culture compared to the control skin. The concentration of all MMPs/TIMP-1 complexes is increased in irradiated skin in coculture with hGF (x2.4 for MMP-1/TIMP-1, 2 times for TIMP-1/MMP-3 and x 2.1 for MMP-9/TIMP-1). The amount of complex is always higher in irradiated skin cultured with hGF compared to the hDF.

hGF prevents elastic network degradation (Figure 2). The irradiated skins co-cultured in the presence of hGF have their collagen and elastic networks less degraded as compared to the skin cultured alone or in the presence of hDF. After 7 days of culture, the fraction of area occupied by the elastic network of the irradiated skin decreased by $56 \%$ as compared to the control skin. The fraction of area of the network decreases by $29 \%$. The co-culture with dermal fibroblasts decreased the degrada- 


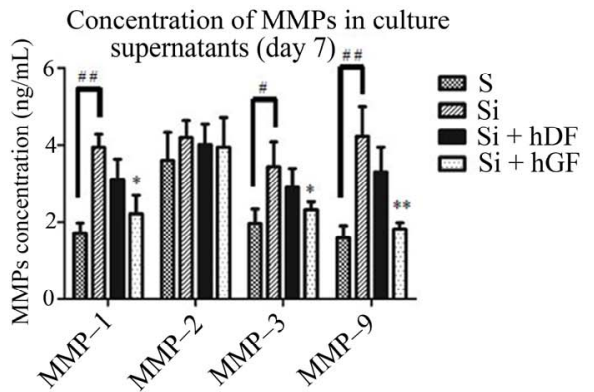

(a)

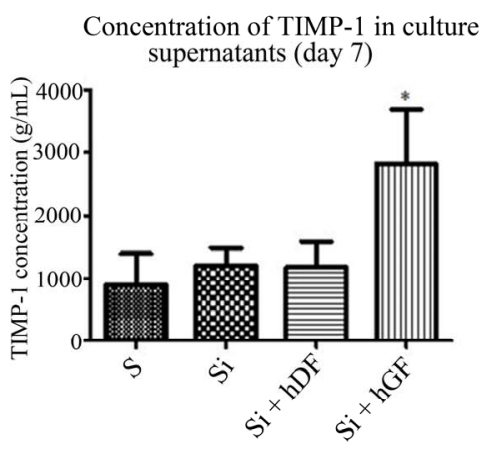

(c)

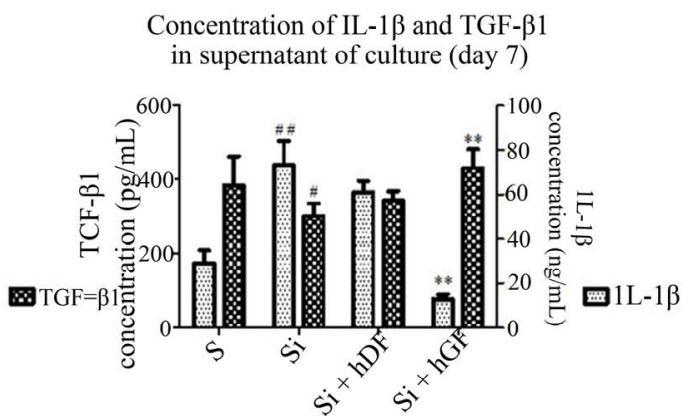

(b)

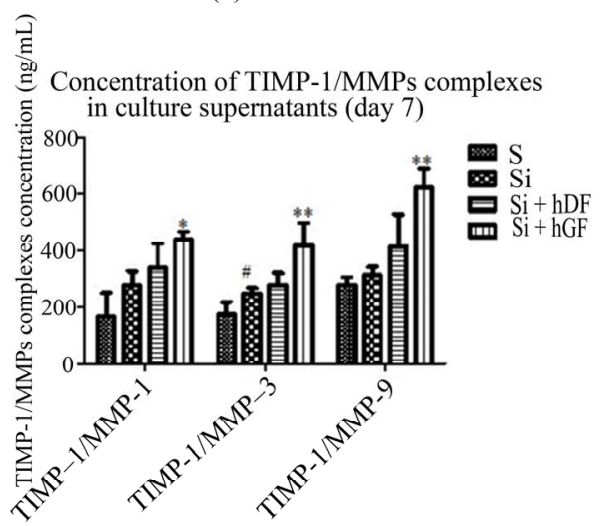

(d)

Figure 1. Effects of gingival fibroblasts and dermal fibroblasts on the irradiated skin at day 7 (ELISA). Figure 1.a: The irradiation of the skin causes an increased synthesis of MMP-1, 3, 9 versus non-irradiated skin. The presence of gingival fibroblast has an effect of inhibiting the activity of MMPs exceeds the dermal fibroblasts. Fig 1.b: The synthesis of TGF- $\beta 1$ is increased in the presence of gingival fibroblast while the synthesis of IL-1 $\beta$ is decreased.Fig1.c, fig1.d. The gingival fibroblast synthesizes more TIMP-1 that the dermal fibroblast. The amount of complex MMP-1, 3.9 / TIMP-1 is formed with upper gingival fibroblasts compared with dermal fibroblasts. \#: Compares the irradiated skin with the non-irradiated skin (\#: p $<0.05, \# \#: \mathbf{p}<0.001) *$ : Compares the irradiated skin in the presence of dermal fibroblast or gingival irradiated with the skin alone $(*: p<0.05 ; * *: p<0.01)$

tion of these macromolecules, but to a lesser extend than the co-culture with hGF. The degradation of the elastic network is of $41 \%$ in the presence of $\mathrm{hDF}$ and $17 \%$ in the presence of hGF. The degradation of the collagen network is of $19 \%$ in co-culture with $\mathrm{hDF}$ and only $9 \%$ in co-culture with hGF.

\subsection{Cellular in Vitro Experiments}

The UVA-irradiated hDF overproduce MMPs. The secretion increases with the radiation dose. The quantifications are performed by zymography and expressed as $\mathrm{Q}=$ surface of bands (S) x 255 grey levels of bands [12]. Thus the amount of MMP-1, compared with the control, produced by $\mathrm{hDF}$ is multiplied by 3.2 (7.5 joules) and by 5 (15 joules) (Figure 3.a). The amount of MMP-3 is, multiplied by 3.6 (7.5 joules) and 5,5 (15 joules) (fig 3.b). The amount of MMP-9 was not detectable without irradiation. It is 36 respectively with irradiation of $\mathrm{hDF}$ at 7.5 joules and increasing to 61 to 15 joules (Figure 3.c).

The hGFm inhibits MMPs produced by irradiated hDF. The overproduction of MMPs is inhibited when the irradiated $\mathrm{hDF}(\mathrm{hDFi})$ were cultured with a conditioned medium of hGF (hGFm). The amount of MMP-1 returns to a basal level for hDFi7.5 and halved for hDFi15. The results are similar for MMP-3. For MMP-9, whatever the irradiation dose, the amount of enzyme detected is reduced by 2 when hDFi are grown in an hGFm. Interestingly, only the hGF conditioned medium, compared with the medium conditioned by the $\mathrm{hDF}$, allows a statistically significant decrease of the amount of MMPs. (Figure 3).

The inhibition is due to the high concentration of TIMP-1 in hGFm and a complex formation (Figure 4). The quantifications are performed by ELISA. The decreasing amount of free enzymes is due to an overproduction by the hGF of the specific tissular inhibitor, the 

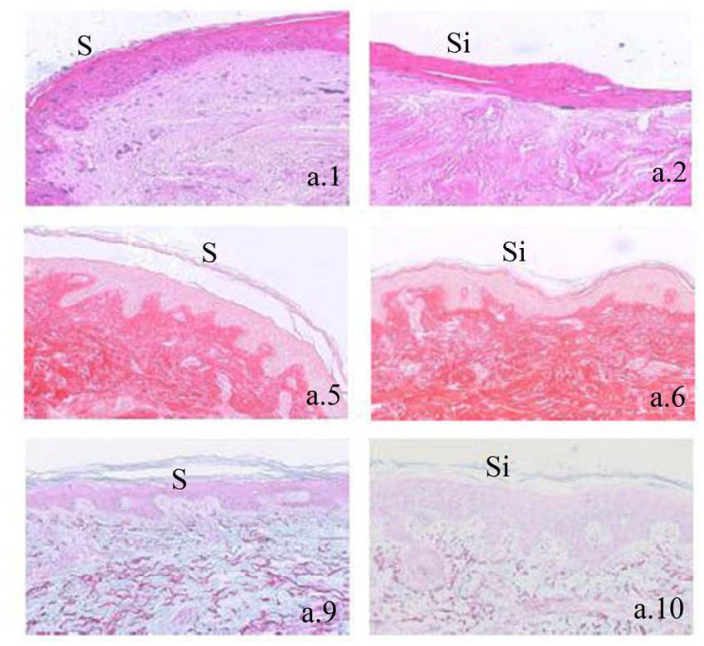

Si

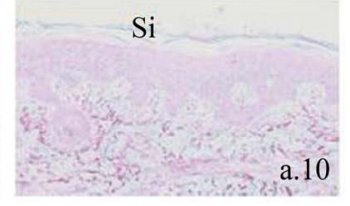

(a)

Area fraction of elastin (\%)

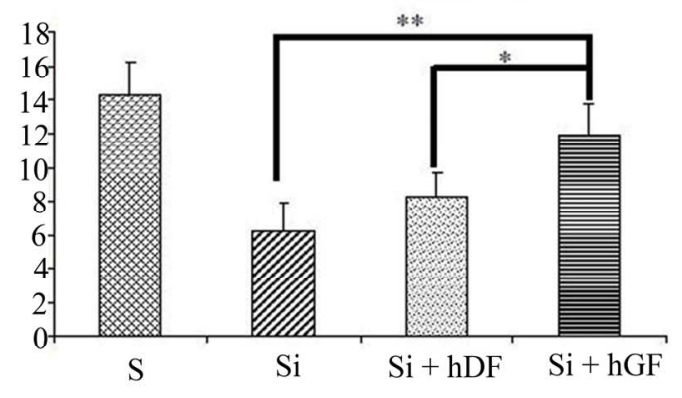

(b)
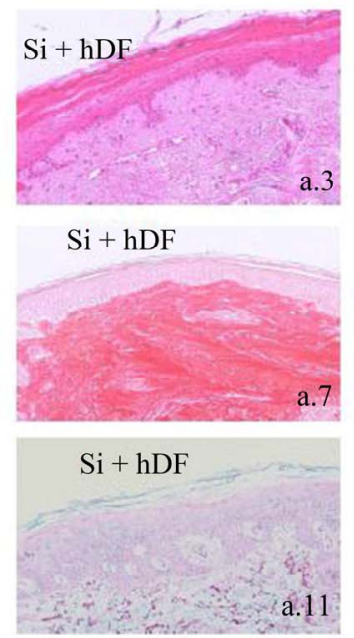

11
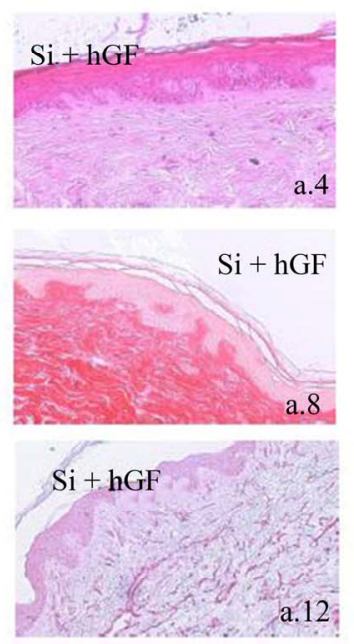

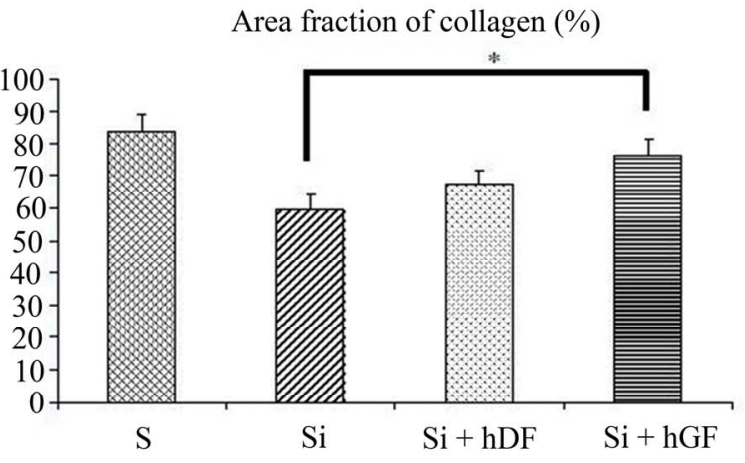

(c)

Figure 2. Preservation of the dermal elastic network by the gingival fibroblast at the seventh day of culture. Figure 2.a: Histological sections of skin (S), irradiated skin (Si), irradiated skin and hDF (Si+ hDF) and irradiated skin and hGF (Si + hGF). Stains were performed: haematoxylin-eosin (cell) (figure 2.a.1 to 2.a.4), sirius red (collagen) (figure 2.a.5 to 2.a.8), orcein (elastic fibers) (figure 2.a.9 to 2.a.10) (original magnification: x125) Figure 2.b: The presence of gingival fibroblast allows an increase in the fraction of area occupied by collagen. Figure 2.c: The presence of hGF allows a preservation of elastic fibers compared to the irradiated skin alone $(p<0.01)$. This preservation by $h G F$ is greater than that induced by the hDF $(\mathbf{p}<\mathbf{0 . 0 5})$

TIMP-1. Indeed the hGFm contains 1.5 times more TIMP-1 than the hDFm. The formation of MMP/TIMP-1 complex is 2-3,4 times greater when irradiated hDF were cultured with hGFm in comparison with irradiated cells without conditioned medium. It should be noted that the irradiated $\mathrm{hDF}$ produces more complex than the nonirradiated $\mathrm{hDF}$. The $\mathrm{hDFm}$ also allows a statistically significant increase in MMP-1/TIMP-1 complex, but only for the highest dose of irradiation. Whatever the amount of experimentation, the MMP/TIMP-1 complex is always much higher in the presence of hGFm than in the presence of $\mathrm{hDFm}$.

Finally we determined in hGFm, the exact amount of the major molecules involved in the remodelling of gingiva and skin (Table 1).

\subsection{Human in Vivo Experiments}

\subsubsection{Studies of Toxicity}

Mutagenic potential of hGFm. The sterility test showed no contamination during the study. No cytotoxic effect was observed and all positive controls performed showed valid ratios $(\mathrm{R})$ above 2.5 . The positive and negative controls showed absolute numbers of revertant colonies comparable to the historical data. No concentration of the hGFm showed a biological significant increase $(\mathrm{R} \geq 2.5)$ of the number of revertant either with or without S9 metabolic activation. No dose response was observed in none of the tested bacterial strains. Based on the results obtained in this study, the hGFm was found to be non mutagenic and non pro-mutagenic.

Skin sensitisation potential of hGFm. No mortality and 


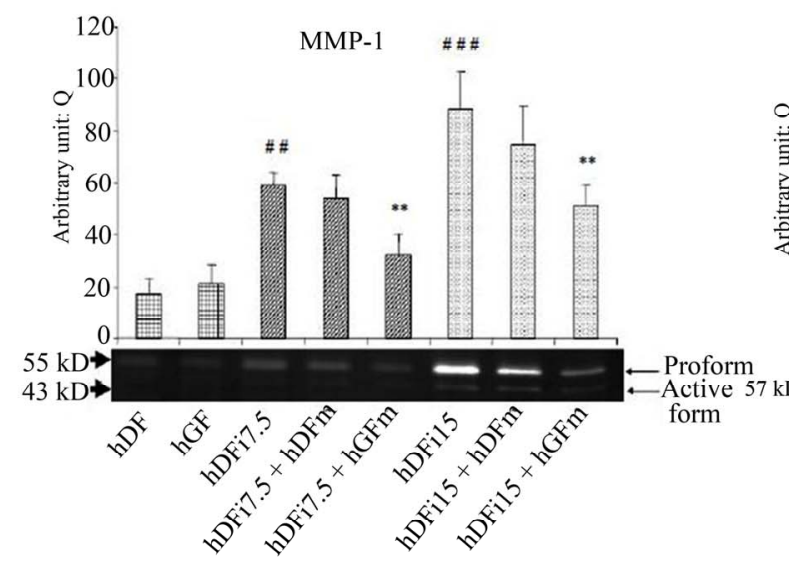

(a)

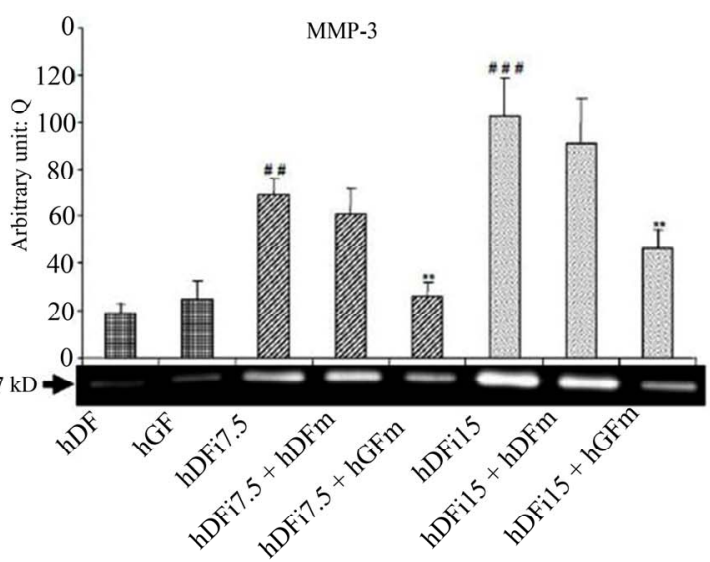

(b)

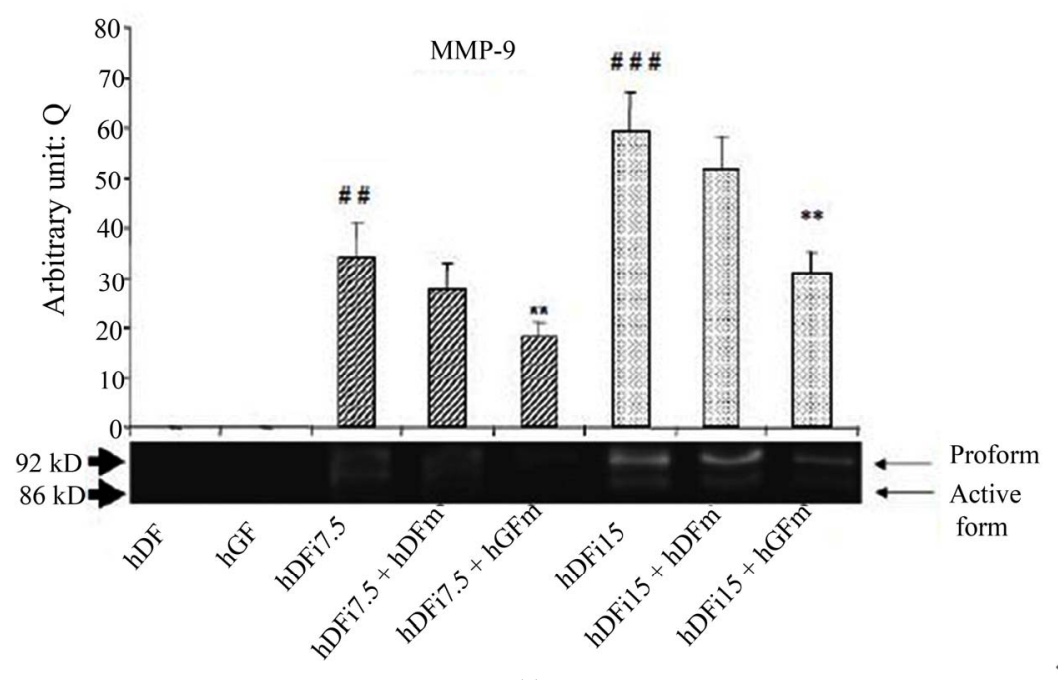

(c)

Figure 3. Effects of conditioned media of HGF (hGFm) and HDF (hDFm) on the activities of MMP-1, 3,9 secreted by the dermal fibroblasts irradiated (hDFi) (Zymogrames). Fig 3.a: The synthesis of MMP-1 is increased in a dose dependent of the irradiation. HDFi culture in the presence of hGFm can decrease the detection of MMP-1 compared to hDFm. Fig 3.b and fig 3.c: The activities of MMP-3 and MMP-9 vary so identical to those of MMP-1. $Q=$ surface of bands (S) $x 255$ gray levels of bands \#: Compares irradiated skin with non-irradiated skin (\#: $p<0.05$, \# \#: $p<0.001) *$ Compares irradiated skin in the presence of conditioned media (hGFm or hDFm) with irradiated skin alone $(*: \mathrm{p}<0.05, * *: \mathrm{p}<0.01)$

Table 1. ELISA of hGFm: The concentration of key molecules involved in tissue remodeling is quantified.

\begin{tabular}{|c|c|c|c|c|c|c|c|c|c|}
\hline MMP1 & MMP2 & MMP3 & MMP9 & TIMP1 & TIMP2 & IL1 $1 \beta$ & IL4 & IL6 & TGF $\beta$ \\
\hline $\begin{array}{l}24 \mathrm{pg} / \mathrm{ml} \\
+/-6\end{array}$ & $\begin{array}{c}31 \mathrm{pg} / \mathrm{ml} \\
+/-7\end{array}$ & $\begin{array}{c}21 \mathrm{pg} / \mathrm{ml} \\
+/-4\end{array}$ & $0 \mathrm{pg} / \mathrm{ml}$ & $\begin{array}{c}86 \mathrm{pg} / \mathrm{ml} \\
+/-17\end{array}$ & $\begin{array}{c}68 \mathrm{pg} / \mathrm{ml} \\
+/-21\end{array}$ & $\begin{array}{c}113 \mathrm{pg} / \mathrm{ml} \\
+/-35\end{array}$ & $\begin{array}{c}321 \mathrm{pg} / \mathrm{ml} \\
+/-67\end{array}$ & $\begin{array}{c}18 \mathrm{pg} / \mathrm{ml} \\
+/-5\end{array}$ & $\begin{array}{c}412 \mathrm{pg} / \mathrm{ml} \\
+/-96\end{array}$ \\
\hline
\end{tabular}

no signs of systemic toxicity were noted in the test and controls animals during the test. Body weight changes of the animals between day 1 and day 6 were comparable to those observed in the corresponding control group animals over the same period. No stimulation index of more than 1.4 was recorded for the three concentrations of the hGFm. No cutaneous reactions were observed at allcon- centrations. In conclusion hGFm must not be classified as a sentitiver in accordance with the criteria for the classification, packaging and labelling of dangerous sub-stances in compliance with the E.E.C. Directive $n^{\circ}$ 67/548, 2001/59 and 99/45. No symbol and risk phrase are required.

Skin irritation test. No dermal reactions (erythema 

and in Vivo Studies

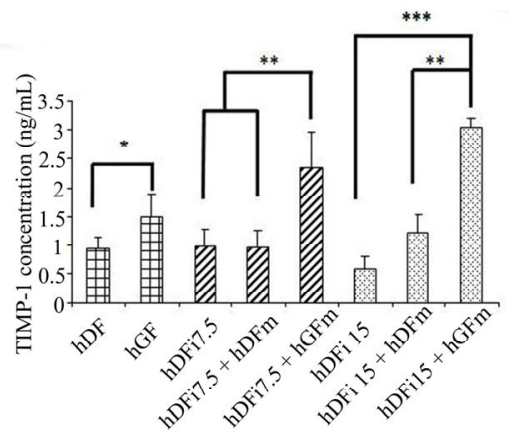

(a)

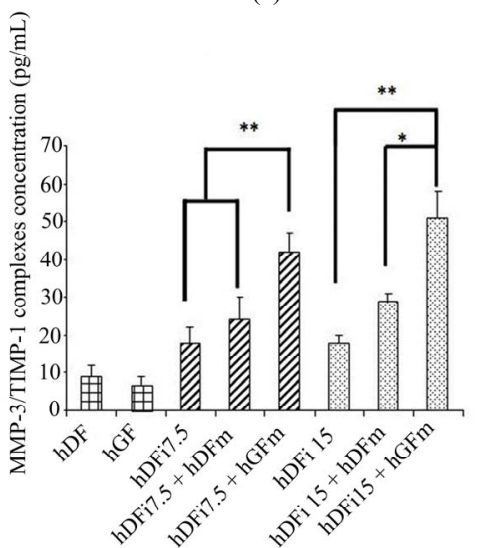

(c)

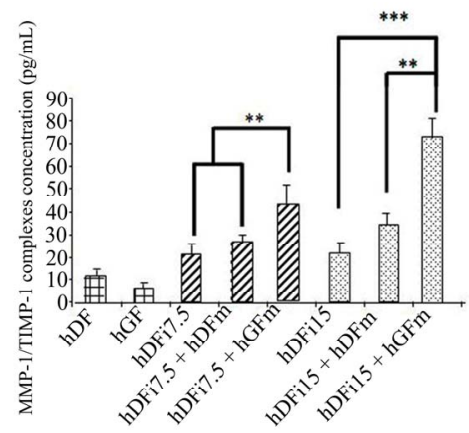

(b)

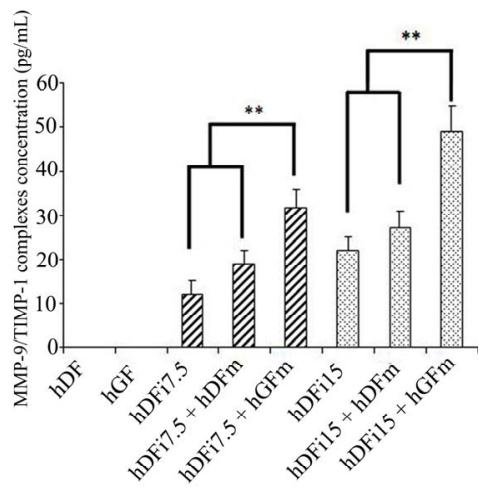

(d)

Figure 4. Concentrations of TIMP-1 and complexes TIMP-1/MMP-1, 3.9 in the culture supernatant of dermal fibroblasts irradiated in the presence of conditioned media or hGFm hDFm (ELISA). Fig 4.a: The hGFm contains more TIMP-1 than the hDFm. The culture of HDFi in the presence of hGFm contains more of TIMP-1 than hDFi cultures in the presence of hDFm or hDFi cultures alone. Fig 4.b, fig 4.c and fig 4.d: This increased synthesis of TIMP-1 is accompanied by an increase in complex formation TIMP-1/MMP-1, 3. 9. Culture in the presence of hDFi hDFm does not change significantly the complex formation of the TIMP-1/MMPs (*: $p<0.05, * *: p<0.01, * * *: p<0.001)$

Table 2. The main results of the clinical study, conducted on humans during a period of six months, are summarized in this table.

\begin{tabular}{|c|c|c|c|c|c|c|}
\hline Parameters & \multicolumn{2}{|c|}{ Before application (day 0) } & \multicolumn{2}{|c|}{ After application (24 weeks) } & \multicolumn{2}{|c|}{ Percentage Change } \\
\hline Number of wrinkles & \multicolumn{2}{|c|}{$97.21+/-39.07$} & \multicolumn{2}{|c|}{$92.36+/-40.68$} & \multicolumn{2}{|c|}{$-4.99 \% *$} \\
\hline Total area $\left(\mathrm{mm}^{2}\right)$ & \multicolumn{2}{|l|}{$7.95+/-4.18$} & \multicolumn{2}{|c|}{$7.11+/-4.06$} & \multicolumn{2}{|c|}{$-10.60 \% \mathrm{NS}$} \\
\hline Total lenght (mm) & \multicolumn{2}{|l|}{$48.57+/-20.06$} & \multicolumn{2}{|c|}{$44.43+/-21.68$} & \multicolumn{2}{|c|}{$-8.52 \% *$} \\
\hline Average lenght (mm) & \multicolumn{2}{|l|}{$0.51+/-0.12$} & \multicolumn{2}{|c|}{$0.48+/-0.14$} & \multicolumn{2}{|c|}{$-5.08 \% *$} \\
\hline Average depth $(\mu \mathrm{m})$ & \multicolumn{2}{|l|}{$58.70+/-9.66$} & \multicolumn{2}{|c|}{$58.82+/-0.19$} & \multicolumn{2}{|c|}{$0.19 \mathrm{NS}$} \\
\hline \multicolumn{2}{|c|}{ Questions } & $\begin{array}{l}\text { I completely } \\
\text { agree }\end{array}$ & I agree & $\begin{array}{l}\text { I do not com- } \\
\text { pletely agree }\end{array}$ & I disagree & $\begin{array}{c}\text { Total } \% \text { of positive } \\
\text { answers }\end{array}$ \\
\hline \multicolumn{2}{|c|}{ The colour is pleasant } & $64 \%$ & $34 \%$ & $0 \%$ & $1 \%$ & $98 \%$ \\
\hline \multicolumn{2}{|c|}{ The texture is pleasant } & $76 \%$ & $24 \%$ & $0 \%$ & $0 \%$ & $100 \%$ \\
\hline \multicolumn{2}{|c|}{ The scent is pleasant } & $53 \%$ & $40 \%$ & $5 \%$ & $2 \%$ & $93 \%$ \\
\hline \multicolumn{2}{|c|}{ The packaging is practical to use } & $53 \%$ & $32 \%$ & $13 \%$ & $2 \%$ & $85 \%$ \\
\hline \multicolumn{2}{|c|}{ The product is pleasant to use } & $75 \%$ & $23 \%$ & $2 \%$ & $0 \%$ & $98 \%$ \\
\hline \multicolumn{2}{|c|}{ The product is easy to apply } & $78 \%$ & $20 \%$ & $2 \%$ & $0 \%$ & $98 \%$ \\
\hline \multicolumn{2}{|c|}{ The skin penetration is rapid } & $56 \%$ & $29 \%$ & $13 \%$ & $2 \%$ & $85 \%$ \\
\hline \multicolumn{2}{|c|}{ The skin does not feel sticky after use } & $53 \%$ & $36 \%$ & $9 \%$ & $2 \%$ & $89 \%$ \\
\hline \multicolumn{2}{|c|}{ The product does not leave a greasy film after use } & $49 \%$ & $35 \%$ & $15 \%$ & $2 \%$ & $84 \%$ \\
\hline \multicolumn{2}{|c|}{ The product induces a feeling of comfort } & $53 \%$ & $42 \%$ & $5 \%$ & $0 \%$ & $95 \%$ \\
\hline
\end{tabular}


and oedema) were observed, on the treated area, whatever the examination times (i.e, 24, 48, 72 hours after the patch removal). The hGFm is non irritant to skin (Psi $=$ 0.0 ) according to the French classification, and must not be classified according to the criteria for the classification, packaging and labelling of dangerous substances in compliance with the E.E.C. Directive $n^{\circ} 67 / 548$, 2001/59 and 99/45. No symbol and risk phrase are required.

Eye irritation test. An ocular conjunctiva reaction has been observed during the study and has been slight and and labelling of dangerous substances in compliance withquickly reversible: only a slight redness noted 1 hour after the hGFm instillation in two animals, and a slight chemosis, noted 1 hour after the hGFm instillation in only one animal. No ocular reaction was noted at the reading time 48 hours. The hGFm must not be classified according to the criteria for the classification, packaging the E.E.C. Directive $n^{\circ} 67 / 548,2001 / 59$ and $99 / 45$. No symbol and risk phrase are required.

At six months the number, length, and the average length of wrinkles are statistically reduced. (Table 2)

After six months of use on wrinkles in the crow's feet (neutral cream containing 5\% hGFm), the number of wrinkles is reduced by $5 \%$, the total length of $8.5 \%$ and the average length of wrinkles by $5 \%$. Although the total area of wrinkles decreased by $10.6 \%$, it is not statistically significant because the deviations are very important. The depth of the wrinkles seems unaffected by the product. In fact, the reduction of the length of wrinkles is statistically verified once a month application. A satisfaction survey showed that $93 \%$ of the users have a favorable opinion of the product.

\section{Discussion}

We have shown in this work that the gingival fibroblast was able to protect the collagen and elastic networks of the skin, ex vivo. Our in vitro experiments permitted to understand the mechanism of this preservation. Indeed, the gingival fibroblast overexpresses TIMP-1, tissue inhibitor of key enzymes responsible for the degradation of the fibrous macromolecules, the MMPs. Thus, the gingival fibroblast behaves, when cultured with skin explants, in the same way than with fragments of arteries $[7,9]$ The TIMP-1 secretion by gingival fibroblasts in coculture causes a complex formation of MMPs/TIMP-1, inhibiting the activity of matrix degradation enzymes. The exceptional remodelling gum are known and largely due to the gingival fibroblast [5]. There is probably no tissue in the body which is subject to attacks as much as the gum: bacterial attacks, chemical, physical, mechanical. These attacks require a permanent adaptation of the gingival fibroblast and explain its responsiveness and its plasticity. This cell is able to migrate, proliferate, synthesize all matrix components and enzymes who are, as well as inhibitors, necessary for the remodelling. The gingival fibroblast also interacts with immune cells such as neutrophils, monocytes-macrophages and lymphocytes. All these abilities turn the gum into a healing model close to the embryo regeneration. As the most obvious clinical evidence, oral healing shows a lack of scar [6,13]. In fact the gingival fibroblast population is very heterogeneous. Some fibroblasts express different cytoskeletal markers such as alpha actin or vimentin reflecting different activities [14]. The protein synthesis [15], the response to cytokines and growth factors [16], the enzyme activities [17], the capacity of proliferation [18] vary among sub-populations of gingival fibroblasts. Moreover, these characteristics are maintained in vitro during passages and cellular ageing [19]. More recently, we demonstrated the existence of a sub-population of progenitor gingival fibroblasts [20]. This sub-population, that may represent $5 \%$ of the total amount of gingival fibroblasts, is extremely important to explain the healing capacity of the gingival tissue.

The second part of this research consists in the study of the effectiveness of a culture medium conditioned by gingival fibroblasts. This medium is very rich in TIMP-1, and is also capable of inhibiting enzymatic activities. The limit of this medium is that it is not renewed by cell secretion and thus depleted of TIMP-1 and other important molecules in remodelling as TGF- $\beta 1$, cytokine synthesis of collagen. Its effectiveness is limited in time, unlike the gingival fibroblast model, which secretes continuously molecules depending on its environment. It was interesting to evaluate this medium on an in vivo study of cosmetology. Indeed the TIMP-1 inhibitor being the key enzymes responsible for degrading macromolecules skin, we tested the middle of gingival fibroblast secretion diluted to $5 \%$ on wrinkles. The lack of toxicity of the medium is checked. Then the culture medium applied daily showed a significant decrease in the length and number of wrinkles. This decrease may seem small in comparison with ex vivo studies. This can be explained both by diluting the product, the need for it to penetrate the skin epithelium and its duration of application. Several prospects now appear in the use of fibroblast against skin ageing. It is clear that using the culture medium rich in and TIMP-1, should be studied in cutaneous injection on animal models.

The use of autologous gingival fibroblast cell therapy opens interesting perspectives. Indeed, the gingival fibroblast is currently used in tracheal reconstruction [21]. This team has shown that only the gingival fibroblasts (in contrast to the dermal and nasal fibroblasts) appears 
compatible with a healthy growth of the tracheal epithelium in the expression of mucin and ion channel activity. Furthermore the coupling of gingival fibroblasts with adipose stem cells further enhances the functionality of the tracheal epithelium [22]. More generally, it seems that the gingival fibroblast, because of its properties, is able to restore in different models, alterations in epithelial and connective tissues. If the effect of TIMP-1 is a key to the understanding of therepair mechanisms of the cell, it is clear that other abilities are implemented in such phenomena. The ability to preserve the elastic network may also be related to properties of protein secretion remodelling of the elastic network [23].The gingival fibroblast secretes more tropoelastin (the precursor of elastic fibers) and fibulin 2 (major component of microfibrils) than periodontal ligament fibroblasts. In addition, the presence of fibroblast progenitors in the population (GMPC) can partly explain the tolerance of different types of tissue recipient's gingival fibroblasts in cellular therapy. Indeed gingival progenitor cells have immunomodulatory properties and improve the inflammatory condition in a model of colitis [24].

\section{Conclusion}

The use of gingival fibroblast and of its secretory products can decrease the activity of the enzymes responsible for the degradation and skin ageing. This preliminary in vivo study of the effectiveness on a conditioned culture medium diluted to $5 \%$ wrinkle is encouraging. It offers new perspectives in the use of an exceptional healing capacity of these cells. They were obtained easily and without sequelae, their ease of cultivation and expansion means that these fibroblast cells appear as interesting cells to be explored in cell therapy against skin ageing.

\section{REFERENCES}

[1] W. Hornebeck, "Down-regulation of tissue inhibitor of matrix metalloprotease-1 (TIMP-1) in aged human skin contributes to matrix degradation and impaired cell growth and survival," Pathologie-biologie, Vol. 51, No. 10, 2003, pp. 569-573. doi:10.1016/j.patbio.2003.09.003

[2] M. Brennan, H. Bhatti, K. C. Nerusu, N. Bhagavathula, S. Kang and G. J. Fisher, et al, "Matrix metalloproteinase-1 is the major collagenolytic enzyme responsible for collagen damage in UV-irradiated human skin," Photochemistry and photobiology, Vol. 78, No. 1, 2003, pp. 43-48. doi:10.1562/0031-8655(2003)078<0043:MMITMC $>2.0$. $\mathrm{CO} ; 2$

[3] G. J. Fisher, S. C. Datta, H. S. Talwar, Z. Q. Wang, J. Varani and S. Kang, et al, "Molecular basis of sun-induced premature skin ageing and retinoid antagonism," Nature, Vol. 379, No. 6563, 1996, pp. 335-339. doi: $10.1038 / 379335 \mathrm{a} 0$
[4] G. J. Fisher, Z. Q. Wang, S. C. Datta, J. Varani, S. Kang and J. J. Voorhees, "Pathophysiology of premature skin aging induced by ultraviolet light," The New England journal of medicine, Vol. 337, No. 20, 1997, pp. 1419-1428.doi:10.1056/NEJM199711133372003

[5] L. Hakkinen, V. J. Uitto and H. Larjava, "Cell biology of gingival wound healing," Periodontol 2000, Vol. 24, 2000, pp. 127-152. doi:10.1034/j.1600-0757.2000.024001127.x

[6] K. Mak, A. Manji, C. Gallant-Behm, C. Wiebe, D. A. Hart and H. Larjava, et al, "Scarless healing of oral mucosa is characterized by faster resolution of inflammation and control of myofibroblast action compared to skin wounds in the red Duroc pig model," Journal of Dermatological Science, Vol. 56, No. 3, 2009, pp. 168-80. doi:10.1016/j.jdermsci.2009.09.005

[7] B. Gogly, A. Naveau, B. Fournier, N. Reinald, E. Durand and C. Brasselet, et al, "Preservation of rabbit aorta elastin from degradation by gingival fibroblasts in an ex vivo model," Arteriosclerosis, thrombosis, and vascular biology, Vol. 24, No. 9, 2007, pp. 1984-1990. doi:10.1161/ATVBAHA.107.140640

[8] A. Naveau, N. Reinald, B. Fournier, E. Durand, A. Lafont and B. Coulomb, et al, "Gingival fibroblasts inhibit MMP-1 and MMP-3 activities in an ex-vivo artery model," Connective tissue research, Vol. 48, No. 6, 2007, pp. 300-308.doi:10.1080/03008200701692461

[9] B. Gogly, B. Fournier, L. Couty, A. Naveau, C. Brasselet and E. Durand, et al, "Gingival fibroblast inhibits MMP-7: evaluation in an ex vivo aorta model," Journal of molecular and cellular cardiology, Vol. 47, No. 2, 2009, pp. 296-303. doi:10.1016/j.yjmcc.2009.04.012

[10] B. Gogly, N. Groult, W. Hornebeck, G. Godeau and B. Pellat, "Collagen zymography as a sensitive and specific technique for the determination of subpicogram levels of interstitial collagenase," Analytical biochemistry, Vol. 255, No. 2, 1998, pp. 211-216. doi:10.1006/abio.1997.2318

[11] A. Naveau, J. J. Lataillade, B. P. Fournier, L. Couty, M. Prat and F. C. Ferre, et al, "Phenotypic Study of Human Gingival Fibroblasts in a Medium Enriched With Platelet Lysate," Journal of periodontology, Vol. 82, No. 4, 2011, pp. 632-641.

[12] J. Y. Beranger, G. Godeau, C. Frances, L. Robert and W. Hornebeck, "Presence of gelatinase A and metalloelastase type protease at the plasma membrane of human skin fibroblasts. Influence of cytokines and growth factors on cell-associated metalloendopeptidase levels," Cell Biology International, Vol. 18, No. 7, 1994, pp. 715-722. doi:10.1006/cbir.1994.1100

[13] J. W. Wong, C. Gallant-Behm, C. Wiebe, K. Mak, D. A. Hart and H. Larjava, et al, "Wound healing in oral mucosa results in reduced scar formation as compared with skin: evidence from the red Duroc pig model and humans," Wound Repair Regen, Vol. 17, No. 5, 2009, pp. 717-729. doi:10.1111/j.1524-475X.2009.00531.x

[14] G. Gabbiani, "The myofibroblast in wound healing and 
fibrocontractive diseases," The Journal of pathology, Vol. 200, No. 4, 2003, pp. 500-503. doi:10.1002/path.1427

[15] A. L. Ejeil, S. Igondjo-Tchen, S. Ghomrasseni, B. Pellat, G. Godeau and B. Gogly, "Expression of matrix metalloproteinases (MMPs) and tissue inhibitors of metalloproteinases (TIMPs) in healthy and diseased human gingival," Journal of periodontology, Vol. 74, No. 2, 2003, pp. 188-195. doi:10.1902/jop.2003.74.2.188

[16] V. M. Bhide, C. A. Laschinger, P. D. Arora, W. Lee, L. Hakkinen and H. Larjava, et al, "Collagen phagocytosis by fibroblasts is regulated by decorin," The Journal of biological chemistry, Vol. 280, No. 24, 2005, pp. 23103-23113.doi:10.1074/jbc.M410060200

[17] C. M. Overall, "Regulation of tissue inhibitor of matrix metalloproteinase expression," Annals of the New York Academy of Sciences, Vol. 732, 1994, pp. 51-64. doi:10.1111/j.1749-6632.1994.tb24724.x

[18] T. M. Hassell and E. J. Stanek, $3^{\text {rd }}$, "Evidence that healthy human gingiva contains functionally heterogeneous fibroblast subpopulations," Archives of Oral Biology, Vol. 28, No. 7, 1983; pp. 617-625. doi:10.1016/0003-9969(83)90010-9

[19] S. Bordin, R. C. Page and A. S. Narayanan, "Heterogeneity of normal human diploid fibroblasts: isolation and characterization of one phenotype," Science New York, Vol. 223, No. 4632, 1984, pp. 171-173.
[20] B. P. Fournier, F. C. Ferre, L. Couty, J. J. Lataillade, M. Gourven and A. Naveau, et al, "Multipotent progenitor cells in gingival connective tissue," Tissue engineering, Vol. 16, No. 9, 2010, pp. 2891-2899.

[21] K. Kobayashi, T. Suzuki, Y. Nomoto, Y. Tada, M. Miyake and A. Hazama, et al, "Potential of heterotopic fibroblasts as autologous transplanted cells for tracheal epithelial regeneration," Tissue Engineering, Vol. 13, No. 9, 2007, pp. 2175-84. doi:10.1089/ten.2007.0008

[22] K. Kobayashi, T. Suzuki, Y. Nomoto, Y. Tada, M. Miyake and A. Hazama, et al, "A tissue-engineered trachea derived from a framed collagen scaffold, gingival fibroblasts and adipose-derived stem cells. Biomaterials," Vol. 31, No. 18, 2010, pp. 4855-4863.

[23] E. Tsuruga, K. Irie and T. Yajima, "Gene expression and accumulation of fibrillin-1, fibrillin-2, and tropoelastin in cultured periodontal fibroblasts," Journal of Dental Research, Vol. 81, No. 11, 2002, pp. 771-775. doi: $10.1177 / 154405910208101110$

[24] Q. Zhang, S. Shi, Y. Liu, J. Uyanne, Y. Shi and S. Shi, et al, "Mesenchymal stem cells derived from human gingiva are capable of immunomodulatory functions and ameliorate inflammation-related tissue destruction in experimental colitis," Journal of Immunology, Vol. 183, No. 12, 2009, pp. 7787-7798. doi:10.4049/jimmunol.0902318 